predictive models to identify 'high risk' readmission patients such as LACE. Historically, in-hospital severity predictive models have been developed for various services and disease states. Some of the most widely used severity measures are the Modified Early Warning Score (MEWS), Glasgow Coma Score (GCS), Acute Physiology, and the American Society of Anesthesiologists (ASA) Score. We wish to compare in-hospital severity scores to predict 30 day readmission. Methods: A retrospective a cohort of patients was assembled who were admitted to two Geisinger hospitals during calendar year 2012. Admissions related to a child's birth, unknown acuity, and in-hospital deaths. Data was obtained during the year preceding the admission and outcomes were assessed up to 30 days post-discharge. Additional data including initial laboratory results, comorbidities, length of stay, and discharge disposition was obtained. For severity scores measured longitudinally during the admission, we selected the initial, last, and worse. A base model of known readmission predictors was built using logistic regression with a flexible specification of continuous measures, and the severity scores were considered individually by forcing them into the model. The net reclassification index (NRI) was used to quantify improvement in prediction. Results: A total of 41,413 admissions met cohort entry criteria (mean age $58.5 \mathrm{y}, 45 \%(18,628)$ male). Readmissions within 30 days occurred $13.3 \%(5,502)$ during 2012 with another $2.9 \%(1,217)$ that died without a readmission. The base model consisting of demographics, comorbidities, discharge disposition, and utilization in past year resulted in an area under the ROC curve (AUC) of 0.710. LACE significantly contributed to the model (AUC $=0.715, \mathrm{NRI}=2.0 \%, \mathrm{OR}=1.12[1.10,1.13]$, $P<0.001)$. The last reported GCS was significantly associated with 30 -day readmission $(\mathrm{OR}=1.05[1.02,1.09])$, however there was no increase in the AUC and the NRI was non-significant. The MEWS was not significantly associated with readmission. Conclusions: The usefulness of in-hospital severity scores was not consistent in their association with readmission. Additional research is needed to understand performance in subgroups and to utilize serial measurements when available.

Keywords: Readmission; Severity

doi:10.3121/cmr.2014.1250.ps1-42

\section{Healthcare Disparities}

D3-1:

HMO Research Network Rural Health Scientific Interest Group (Rural Health-SIG): Highlights from Four Member Sites and Opportunities for Collaboration

David McClure'; Jeffrey Van Wormer'; Stephen Waring²; Thomas Elliot²; Lisa Bailey-Davis ${ }^{3}$; Laurel Copeland ${ }^{4}$

${ }^{1}$ Marshfield Clinic Research Foundation; ${ }^{2}$ Essentia Institute of Rural Health; ${ }^{3}$ Geisinger Health System; ${ }^{4}$ Scott \& White Healthcare

Background/Aims: Compared to their urban counterparts, rural residents have persistent health disparities and remain medically underserved. However as shown last year by the Rural Health SIG, many diseases and health conditions strongly impact rural and urban dwellers (Copeland et al, HMORN 2013 PS3-47). To put this in perspective, we describe the member populations and highlight the research specialties from four of seven Rural Health-SIG sites with a substantial number of members living in rural areas. Methods: We identified member population sizes, areas of scientific emphasis, and research resources for Essentia Institute of Rural Health (EIRH), Geisinger Center for Health Research (GCHR), Marshfield Clinic Research Foundation (MCRF), and Scott \& White Center for Applied Health Research (SWR). Sources were VDW documentation, HMO Research Network Members-Areas of Scientific Emphasis Based on Self-Report (2011/2012), and publicly available websites for each of the sites. Results: Currently, there are over 1 million members in 2012 VDW enrollment (EIRH: 565,603; GCHR: 310,834; MCRF: 195,182; SWR: 94,351) of which at least half live in rural areas and at least $30 \%$ receive government funded health insurance assistance (Medicare, Medicaid, or state subsidies). Collectively, we have ongoing research activity in all areas of HMORN scientific interest. Areas of significant emphasis (multiple projects per year) for at least two sites include: obesity, active living, healthy eating; dissemination science and translation of research into practice; patient decision-making and health literacy; quality improvement and health care delivery; genetics, genomics, bio-repositories; health informatics; heart/ vascular, lung and blood diseases; neurological disorders; and aging. Moreover, all four sites reported significant emphasis in community engagement and community-based participatory research. In comparison to the 11 other, primarily urban HMORN member sites, eight reported some emphasis in this area and none reported significant emphasis. Patientreported outcome capability is also rapidly developing at Rural Health SIG sites in response to deployment of electronic medical record systems and the quest to achieve Accountable Care Organization status. Conclusions: Rural Health SIG Investigators have broad expertise in many areas of scientific interest within the HMORN that apply to improving the health of rural and urban residents. We welcome invitations to collaborate.

Keywords: Healthcare disparities; Rural health

doi:10.3121/cmr.2014.1250.d3-1

D3-2:

Forecasting Disease Burden and Health Utilization in Uninsured Populations in California Health Insurance Exchange Pricing Regions

Tuan Dinh ${ }^{1}$

\section{${ }^{1}$ Kaiser Permanente Northern California}

Background/Aims: Starting in January 2014, millions of currently uninsured Californians will be able to purchase health insurance on the California Health Insurance Exchange (HIE). For planning purposes, it is important to estimate disease burden and demands for services in uninsured populations. The Archimedes Model is a trial-validated, clinically detailed simulation model of human physiology, disease progression, and healthcare delivery. The Archimedes Model synthesizes evidence from hundreds of data sources to create virtual patients that are representative of the US population, in terms of demographic distribution, disease progression, clinical outcomes, patient and provider behaviors; office visits and hospital admissions; tests and treatments; care delivery protocols; compliance; and costs. Kaiser Permanente and Archimedes collaborated to develop a capability based on the Archimedes Model to forecast disease burden and healthcare utilization in uninsured populations for each of the 18 California Health Insurance Exchange pricing regions. Methods: We used data from 20 diverse California and US surveys and databases as inputs into the Archimedes Model to create realistic individuals that closely match the uninsured populations in each pricing region, with respect to demographics, socioeconomic status, and medical conditions. Within the Archimedes Model, the relationships between biomarkers, medication adherence and usage, disease progression, clinical outcomes, care processes and healthcare utilizations are well-established and carefully validated against many independent datasets. We use the Archimedes Model to combine datasets with overlapping information and to fill in the missing information for uninsured Californians, then forecast the clinical outcomes and health utilizations in the next 10 years for this population as well as the benefits and costs of different prevention and disease management strategies. Results: We created 19 datasets that are representative of the pricing regions on the Health Insurance Exchange and cover a wide range of outcomes and conditions, including diabetes, cardiovascular diseases, cancers, COPD, asthma, childbirths and mental health. Conclusions: Health plans and insurers can use the information provided by the datasets for resource allocation and capacity planning. Local officials will able to use these datasets measure today's health of their communities and forecast tomorrow's health.

Keywords: Uninsured; Healthcare utilization doi:10.3121/cmr.2014.1250.d3-2

D3-3:

Differing Quality or Differing Expectations: Should One Adjust for Patient Race/Ethnicity when Assessing Patient Satisfaction?

Sukyung Chung ${ }^{1}$; Latha Palaniappan ${ }^{1}$; Edith Gamboa ${ }^{1}$; Hal Luft ${ }^{1}$

${ }^{1}$ Palo Alto Medical Foundation Research Institute

Background/Aims: The literature indicates Asians rate their health care experience lower than do non-Hispanic whites (NHWs). Cultural norms in rating similar experience may contribute to this, but before any adjustment, one should make sure differing ratings do not reflect true quality differences. We examined whether adjustment for the race/ethnicity $(\mathrm{R} / \mathrm{E})$ of survey 
respondents is warranted in assessing patient satisfaction across providers. Methods: We used 2011-12 patient satisfaction data from a large ambulatory care organization (116,516 surveys for 1,022 providers). Surveys were mailed to patients after randomly selected clinic visits. Using the scoring scheme used by survey organization, answers to each question (on a 5-point Likert scale) were converted to a $0-100$ scale. The overall score for each visit was an average of 6 section scores: Access, Moving through the visit, Nurse/ assistant, Care provider, Personal issues, and Overall assessment. The overall visit score was aggregated at provider, clinic and region levels. We focus on overall and section scores across 4 regions of the organization. Results: R/E differences were substantial, from 90.7 (NHW) to 85.2 (Asian). Regional scores ranged from 88.2 (region A) to 90.1 (region D). The ranking was not consistent, however, within R/E groups; Region A scored highest among Latino and 2nd among NHW patients, while region D scored lowest among Latino and 3rd among NHW patients. True differential treatment by R/E should be most apparent in "Care provider" and "Personal issues", and less so in "Access" and "Moving through the visit". In fact, the R/E difference (NHW vs. Asian) was smallest for "Care provider" (4.3) and largest for "Moving through the visit" (7.0) scores. R/E differences were much less pronounced if the top 2 Likert categories were combined. Conclusions: Patient satisfaction ratings are influenced by patient $\mathrm{R} / \mathrm{E}$ in that similar experiences may not be rated equally. When R/E composition varies across providers or regions, scores need to be adjusted to account for this effect or scales less sensitive to differential 'top box' scoring may be appropriate. Keywords: Patient satisfaction; Cultural difference

doi:10.3121/cmr.2014.1250.d3-3

D3-4:

The Intersection of Ageism and Heterosexism: LGBT Older Adults' Perspectives on Aging-in-Place

Jennifer Boggs ${ }^{1}$; Jennifer Dickman Portz'; Leslie Wright ${ }^{1}$; Diane King²; Cathy Grimm³ ${ }^{3}$ Jessica Retrum ${ }^{4}$; Wendolyn Gozansky ${ }^{1}$

${ }^{1}$ Kaiser Permanente Colorado; ${ }^{2}$ University of Alaska Anchorage; ${ }^{3}$ Jewish Family Service; ${ }^{4}$ Metropolitan State University of Denver

Background/Aims: Successful aging in place models promote independence, include older adults in decision-making, create an environment of personal and physical safety, facilitate social support, and provide services to enhance the health and quality of life of older adults within the communities in which they live. The applicability of aging in place models for disenfranchised groups is an important consideration. The current study, undertaken as part of a larger community-research partnership, examined the adequacy of this approach with older LGBT adults in a metropolitan neighborhood. Methods: This qualitative study used focus groups $(\mathrm{n}=14)$, interviews $(\mathrm{n}=29)$, and a town hall $(\mathrm{n}=30)$ to assess barriers and supports for LGBT persons to age in place. Results: Most of the 73 participants identified as lesbian or gay, were aged 50-69, and lived with a partner. Discrimination and dual stigmatization (ageism plus heterosexism) emerged as cross-cutting themes that negatively impacted the aging in place categories of healthcare, housing, social support, home assistance and legal services. Establishing welcoming social spaces, disseminating knowledge resources, and promoting selfadvocacy were suggested solutions. Conclusions: This study provides a unique contribution to knowledge about the needs of LGBT older adults pertaining to aging in place. While resilience and coping skills developed at younger ages during the "coming out" process can continue to be leveraged in later years, LGBT seniors who are less "out" are likely particularly vulnerable to the challenges presented by the intersection of ageism and heterosexism. This research offers recommendations for tailoring current aging in place models to better suit the needs of LGBT older adults.

Keywords: Aging in place; Community-research partnership

doi:10.3121/cmr.2014.1250.d3-4

D3-5:

The Role of Community Engagement in Increasing Translational Health Research Participation by Hispanics in the WWAMI Region

Leo Morales ${ }^{1}$; Nora Coronado

${ }^{1}$ Group Health Research Institute
Background/Aims: Increasingly, community engagement is used to increase the participation by minority communities in health research and to address health disparities. The CDC defines community engagement as "the process of working collaboratively with and through groups of people affiliated by geographic proximity, special interest, or similar situations to address issues affecting the well-being of those people". The goal of the Institute for Translational Health Science's (ITHS) Hispanic Community Outreach Program (HCOP) is to increase participation by Hispanics in translational health research conducted by ITHS in the WWAMI region (Washington, Wyoming, Alaska, Montana, Idaho). Methods: The HCOP has conducted outreach to investigators, community-based organizations (CBOs) and clinics serve Hispanics in the WWAMI region since August 2012. The HCOP's outreach activities have included phone calls, site visits, and participation in community events and conferences, resulting in a growing network of potential research partners. This network has evolved over time through personal contacts and referrals, reflecting the importance of interpersonal relationships and trust when engaging communities. To track its work, the HCOP maintains a database with the following elements: name, address, phone, email, agency affiliation and department, title, degrees, region, and level of engagement (LOE). The LOE is scored on a graded scale of 1-5 where 1 indicates a minimal LOE and 5 indicates a high LOE. Results: To date, the HCOP has made contact with over 840 stakeholders of whom $42 \%$ were investigators; $54 \%$ were in CBOs; and $6 \%$ were in government health agencies. The majority of HCOP's initial contacts had LOE scores in the 1-2 level. Over time, LOE scores have increased, including one partnership that received grant funding to disseminate a diabetes selfmanagement program in Spanish. The HCOP was also awarded a NIH small conference grant for a regional conference on Latino health. In addition, the HCOP has awarded pilot funding for community engaged research. Conclusions: Community engagement appears to be an effective strategy for increasing the participation of Hispanics in translational health research. More research is needed to develop tools and methods for assessing the effectiveness of community engagement and its impacts on the health of communities.

Keywords: Community engagement; Hispanic

doi:10.3121/cmr.2014.1250.d3-5

PS1-41:

Racial Disparities in Congestive Heart Failure: Age, Site of Care, and 30-day Readmission

Jia $\mathrm{Pu}^{1}$; Joshua Thorpe ${ }^{2}$; Korey Kennelty ${ }^{3}$; Betty Chewning ${ }^{4}$

${ }^{1}$ Palo Alto Medical Foundation Research Institute; ${ }^{2}$ University of Pittsburgh; ${ }^{3}$ William S. Middleton Memorial Veterans Hospital; ${ }^{4}$ University of Wisconsin

Background/Aims: Racial disparities of 30-day readmission rates have been found in Medicare beneficiaries with congestive heart failure (CHF); however, racial disparities in the younger age groups remained unknown. Further, the association between patient demographics, site of care and 30-day readmissions are understudied. Our study aims to identify racial disparities in CHF patients across age groups, site of care and 30-day readmission rate, and to explore potential contributing variables to the racial disparity in CHF. Methods: This study used the 2009 Florida State Healthcare Cost and Utilization Project State Inpatient Databases (HCUPSID). Patients 18 years or older, hospitalized with a primary diagnosis of CHF were included in this study. Multi-level modeling examined if racial disparities in risk-adjusted 30-day readmission rate could be explained by patients' characteristics (age, gender, insurance, household income, rurality, length of stay, discharge status) and hospital characteristics (whether the site of care was a minority-serving hospital at their initial admission, defined as the top $10 \%$ of hospitals who served the highest proportion of non-white patients). Results: Racial disparities existed in CHF patients' age at discharge, site of care, and risk-adjusted 30-day readmission rate. African American CHF patients as a group were hospitalized on average 14 years younger than Whites, and 10 years younger than Hispanics (AA: 63 vs. White: 77 vs. Hispanics: $73, P<0.001)$. Overall, Hispanic patients had the highest readmission rate (Hispanics: 27.7\%, AA: 25.9\%, White: $24 \%$ ). Higher readmission rates were associated with race of minority, Medicare and Medicaid program enrollment, lower income, discharge status, minorityserving hospital, and younger age (age $<55: 29 \%$, age 55-64: $27 \%$, age $65-74$ : 25\%, age $75-84: 24 \%$, age $>84: 22 \% . P<0.001)$. This age trend remained in 